\title{
Resonant Characteristics of Triple-Mode Dielectric Resonators
}

\author{
Jae-Yoon Myung ${ }^{1} \cdot$ Sang-Won Yun ${ }^{1, *} \cdot$ Namshin Park $^{2} \cdot$ Donyong Lee ${ }^{2}$ Byungchul Kim ${ }^{2}$. \\ Jeonghee $\mathrm{Won}^{2} \cdot$ Inho $\mathrm{Na}^{2} \cdot$ Geonho $\mathrm{Jang}^{2} \cdot \mathrm{Xu}-$ Guang Wang ${ }^{2}$
}

\begin{abstract}
This paper discusses the resonant characteristics, such as the resonant frequencies and Q factors, as well as tuning methods of the resonant frequency of the triple-mode dielectric resonators, for their possible application to compact bandpass filters for mobile communication systems. The resonators include spheres and cylindrical rods that can be applied to compact bandpass filters. These high $Q$ ceramic resonators can replace the conventional coaxial type resonators, which are large sized and also have relatively low $Q$ values. Both simulated and measured results show that the Q values of the sphere and rod reach 27,000 and 24,000, respectively. Decoupling of degenerate modes is also briefly discussed.
\end{abstract}

Key Words: Dielectric Resonator, Dielectric Rod, Dielectric Sphere, High Q, Triple-Mode.

\section{INTRODUCTION}

Mobile communication systems support multiband/multimode operation, which requires several bandpass filters and which becomes inevitably bulky with conventional cavity type resonators. The base station as well as the remote radio head now requires compact multiband bandpass filters with high performance. This high performance and compactness of the bandpass filters can be accomplished by replacing conventional cavity resonators with ceramic resonators, which have been widely used in satellite communication systems. This paper examines the use of these ceramic resonators in mobile communication systems.

The conventional dielectric resonator used for satellite transponders is a dielectric disk in which the two resonant modes are degenerate. A bandpass filter made with this resonator has good stability against temperature variations and has been applied to many satellite communication systems [1
-3]. In 1997, a triple-mode dielectric rod resonator was proposed to reduce the size of the bandpass filter [4]. Since this resonator has triple modes, a 3-pole bandpass filter can be designed by one triple-mode resonator. Using two triplemode resonators, a 6-pole elliptic function bandpass filter was successfully demonstrated for a satellite transponder in [5].

The ceramic resonators analyzed in this paper are dielectric spheres and rods in which triple modes exist. The resonant characteristics are essential to the design of bandpass filter. The electromagnetic fields in these resonators are well known [6]; therefore, only their resonant characteristics, including the tuning method, will be discussed.

\section{TRIPLE-Mode RESONATORS}

Triple modes can also be supported in a dielectric cube. However, because of the $Q$ values, two types-sphere and rod-will be discussed. The dielectric resonator must be

Manuscript received November 7, 2013 ; Revised February 4, 2014 ; Accepted February 17, 2014. (ID No. 20131107-045J)

${ }^{1}$ Department of Electronic Engineering, Sogang University, Seoul, Korea.

${ }^{2}$ KMW Inc., Hwasung, Korea.

"Corresponding Author: Sang-Won Yun (e-mail: swyun@sogang.ac.kr)

This is an Open-Access article distributed under the terms of the Creative Commons Attribution Non-Commercial License (http://creativecommons.org/licenses/by-nc/3.0) which permits unrestricted non-commercial use, distribution, and reproduction in any medium, provided the original work is properly cited.

(C) Copyright The Korean Institute of Electromagnetic Engineering and Science. All Rights Reserved. 


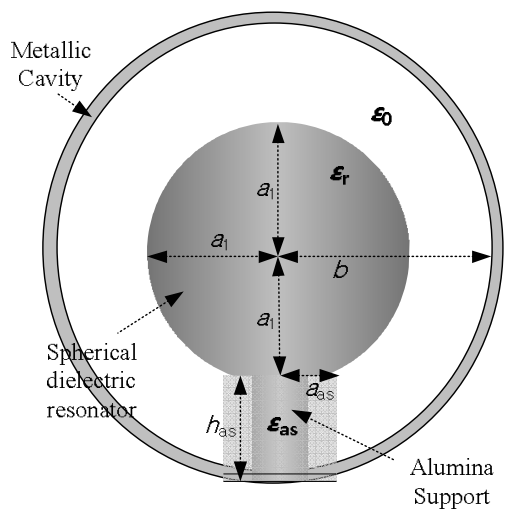

Fig. 1. A spherical dielectric resonator with alumina support in a metallic enclosure.

placed inside the metallic housing or cavity; therefore, the dimensions of the metallic cavities and mounts for the dielectric sphere and rod are determined in such a way that they do not affect the resonant characteristics of dielectric resonators very much.

\section{The Spherical Dielectric Resonator}

As illustrated in Fig. 1, a spherical dielectric resonator with radius $a_{1}$ is placed at the center of a spherical metallic cavity with radius of $b$ by an alumina support with permittivity of $\varepsilon_{\text {as }}$, height $h_{\text {as }}$, and radius $a_{\text {as. }}$. This dielectric sphere can support three orthogonal degenerate modes: $\mathrm{TE}_{01 \delta \mathrm{X}}$, $\mathrm{TE}_{01 \delta \mathrm{Y}}$, and $\mathrm{TE}_{01 \delta \mathrm{Z}}$ along the $\mathrm{x}^{-}, \mathrm{y}^{-}$, and $\mathrm{z}$-direction, respectively. The electric field configurations of the three modes plotted in Fig. 2 show that the three resonant electric fields have a rotational symmetry. The bandpass filter should be designed by splitting the resonant frequencies in such a way that the three resonant modes constitute three distinct reflection zeroes within the passband.

We can introduce the circumferential chamfering of the sphere as shown in Fig. 3, so that the resonant frequency of the $\mathrm{TE}_{01 \delta \mathrm{Z}}$ mode is shifted upward, while that of the other two modes are not affected. The sphere surface along the $\mathrm{z}$-axis is chamfered, so the radius is reduced from $a_{1}$ to $a_{2}$ as shown in Fig. 3, for the fine frequency tuning of $\mathrm{TE}_{01 \delta \mathrm{Z}}$ mode without appreciably affecting the other two modes. The bottom of the dielectric sphere is also chamfered slightly in order to mount it onto the hollow cylindrical alumina support. This bottom surface chamfering can also be used for fine control of the resonant frequencies of the $\mathrm{TE}_{01 \delta \mathrm{X}}$ and $\mathrm{TE}_{01 \delta \mathrm{Y}}$ modes. The dimension of the metal cavity is set as $b=2 a_{1}$ in order not to affect the resonant frequencies of the dielectric resonator. The modified resonator in Fig. 3 has the following dimensions for HFSS simulation at $800 \mathrm{MHz}$, using the equation given by Kajfez and Guillon [7]:

$a_{1}=29.4 \mathrm{~mm}, a_{2}=27.3 \mathrm{~mm}, a_{3}=22.5 \mathrm{~mm}$, and $b=44.76$ $\mathrm{mm}$ and the dimensions of the alumina support in Fig. 3 is given as

$$
\varepsilon_{\mathrm{ras}}=9, a_{\mathrm{as}}=13 \mathrm{~mm}, h_{\mathrm{as}}=20 \mathrm{~mm}, t_{\mathrm{as}}=5 \mathrm{~mm} \text {. }
$$

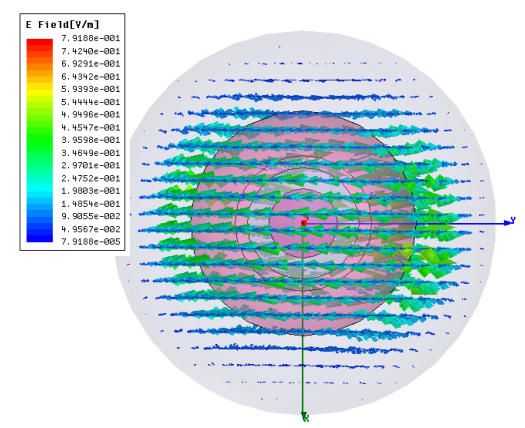

(a)

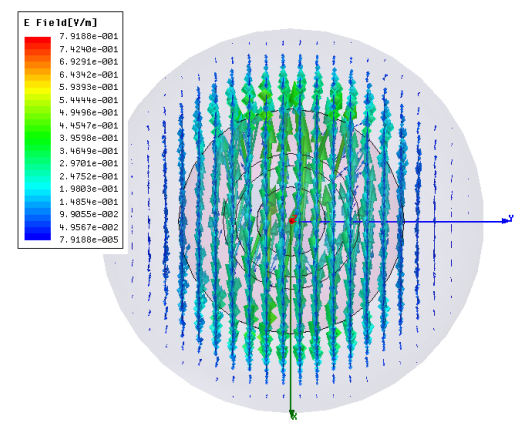

(b)

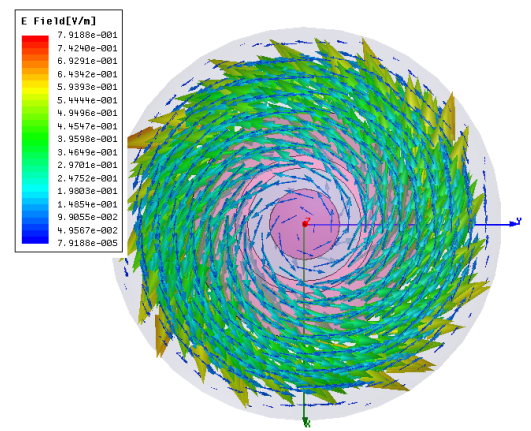

(c)

Fig. 2. Top view of E-field distribution along the axes: (a) $\mathrm{TE}_{010 \mathrm{X}}$ at $845.66 \mathrm{MHz},(\mathrm{b}) \mathrm{TE}_{01 \delta \mathrm{Y}}$ at $845.69 \mathrm{MHz}$, and (c) $\mathrm{TE}_{018 \mathrm{Z}}$ at $852.76 \mathrm{MHz}$.

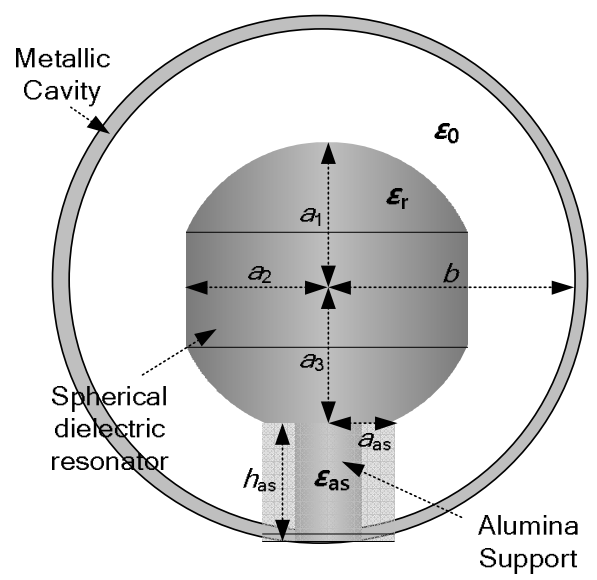

Fig. 3. Chamfered spherical resonator.

The corresponding resonant frequencies are $f_{1}\left(\mathrm{TE}_{01 \delta \mathrm{X}}\right)=$ 


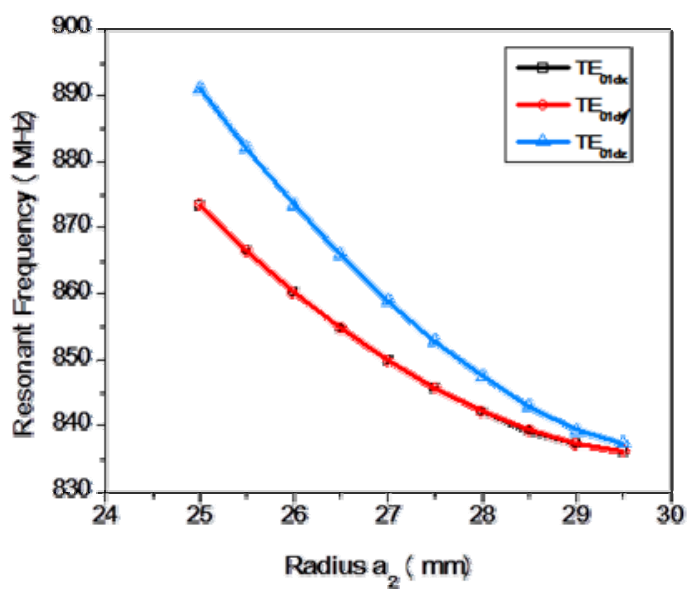

Fig. 4. The resonant frequency change due to circumferential chamfering in Fig. 3.

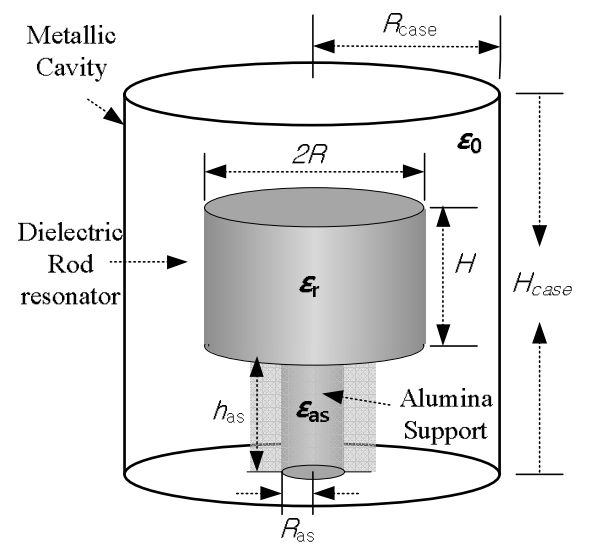

Fig. 5. Dielectric rod located inside the metallic cavity.

845.66 MHz, $f_{2}\left(\mathrm{TE}_{01 \delta \mathrm{Y}}\right)=845.69 \mathrm{MHz}$, and $f_{3}\left(\mathrm{TE}_{01 \delta \mathrm{z}}\right)=$ $852.76 \mathrm{MHz}$. The relative permittivity of spherical dielectric resonator is 46 and the calculated unloaded $Q$ value, $Q_{\mathrm{U}}$, is 19600. The chamfering of the circumferential area affects the resonant frequency $f_{3}\left(\mathrm{TE}_{01 \delta \mathrm{Z}}\right.$ mode $)$ as shown in Fig. 4, while the effect of the chamfering at the bottom of the sphere is not pronounced.

\section{Dielectric Rod}

The cylindrical dielectric rod shown in Fig. 5 can also support triple resonant modes. However, in this structure, only two $\mathrm{TE}_{016 \mathrm{X}}$ mode and $\mathrm{TE}_{01 \delta \mathrm{Y}}$ mode are degenerate. The third mode, $\mathrm{TE}_{018 \mathrm{Z}}$ mode, which has a very similar electric field distribution along the cross section, has different resonant frequencies depending on the length $H$. By setting the diameter of the rod nearly equal to the length of the rod, $H$, one can observe that the resonant frequency of the $\mathrm{TE}_{01 \delta \mathrm{Z}}$ mode is located very close to that of two degenerate modes ( $\mathrm{TE}_{01 \delta \mathrm{X}}$ and $\mathrm{TE}_{01 \delta \mathrm{Y}}$ modes). The electric field distributions of three modes are plotted in Fig. 6; they are not much different from those of sphere resonators. The three modes are orthogonal to each other and designated as the $\mathrm{TE}_{018 \mathrm{X}}$,

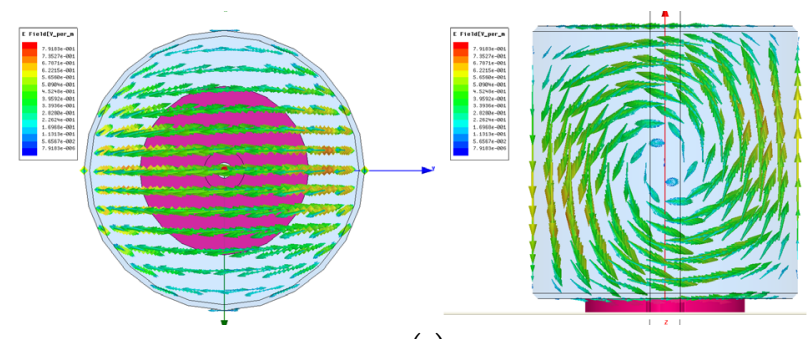

(a)

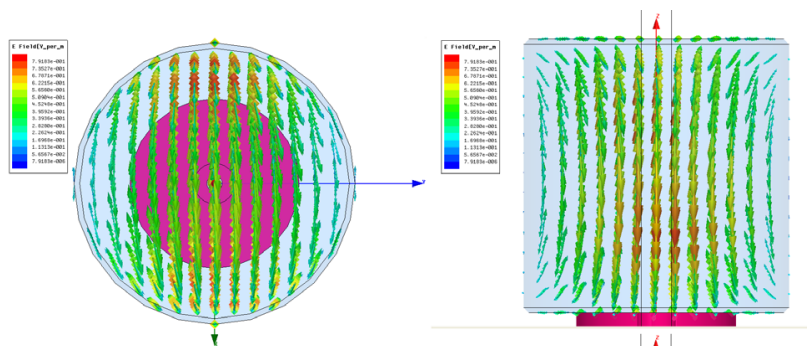

(b)
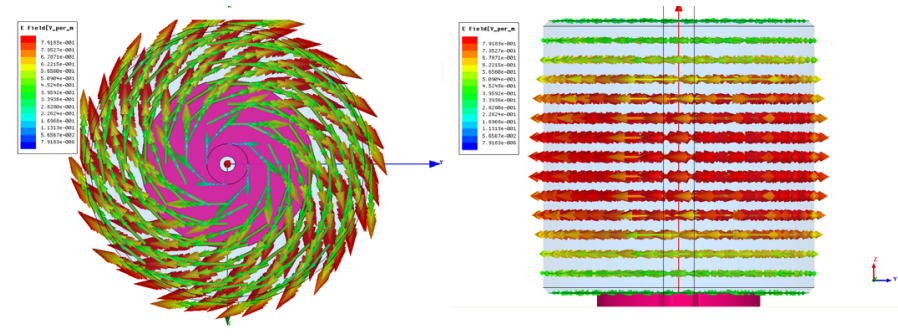

(c)

Fig. 6. E-field distributions of three orthogonal modes: (a) $\mathrm{TE}_{01 \delta \mathrm{X}}$ at $842.70 \mathrm{MHz},(\mathrm{b}) \mathrm{TE}_{01 \delta \mathrm{Y}}$ at $842.85 \mathrm{MHz}$, and (c) $\mathrm{TE}_{01 \delta \mathrm{Z}}$ at $846.85 \mathrm{MHz}$.

Table 1. Resonant frequency $(\mathrm{MHz})$ variations as a function of rod length $H$

\begin{tabular}{cccccccc}
\hline \multirow{2}{*}{$\begin{array}{c}H \\
(\mathrm{~mm})\end{array}$} & \multicolumn{3}{c}{$H / R=0.49$} & & \multicolumn{3}{c}{$\mathrm{H} / \mathrm{R}=0.51$} \\
\cline { 2 - 3 } \cline { 7 - 8 } & $\mathrm{TE}_{01 \delta \mathrm{X}}$ & $\mathrm{TE}_{01 \delta \mathrm{Y}}$ & $\mathrm{TE}_{01 \delta \mathrm{Z}}$ & & $\mathrm{TE}_{01 \delta \mathrm{X}}$ & $\mathrm{TE}_{01 \delta \mathrm{Y}}$ & $\mathrm{TE}_{01 \delta \mathrm{Z}}$ \\
\hline 42.5 & 991.02 & 991.07 & 995.73 & & 968.33 & 968.36 & 966.94 \\
45 & 936.45 & 936.72 & 940.74 & 914.34 & 914.40 & 913.17 \\
47.5 & 886.78 & 886.81 & 890.93 & 866.53 & 866.63 & 864.82 \\
50 & 842.95 & 843.09 & 846.56 & 823.33 & 823.37 & 821.59 \\
52.5 & 803.00 & 803.08 & 806.24 & 784.26 & 784.31 & 782.53 \\
55 & 766.22 & 766.25 & 769.37 & & 749.52 & 749.67 & 747.49 \\
57.5 & 732.98 & 732.99 & 735.85 & & 716.04 & 716.09 & 714.44 \\
60 & 702.67 & 702.69 & 705.40 & 687.08 & 687.18 & 685.06 \\
\hline
\end{tabular}

$\mathrm{TE}_{01 \delta \mathrm{Y}}$, and $\mathrm{TE}_{01 \delta \mathrm{Z}}$ modes, respectively, as shown in Fig. 6. Therefore, we simulate the variation of resonant frequency at $850 \mathrm{MHz}$ range as a function of rod radius and rod length as shown in Table 1. The resonant frequency of the $\mathrm{TE}_{018 \mathrm{Z}}$ mode is greatly affected when $H / R$ is less or greater than 0.5 . In the simulation, the dimension of the outer metallic cavity was determined in such a way that the triple resonant frequencies were not affected. 


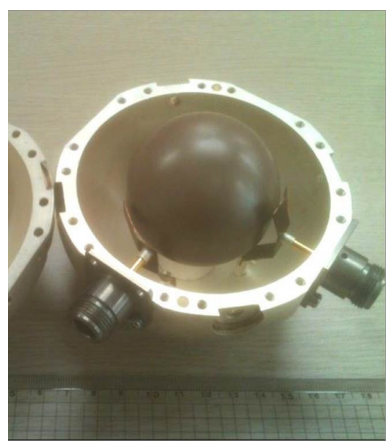

(a)

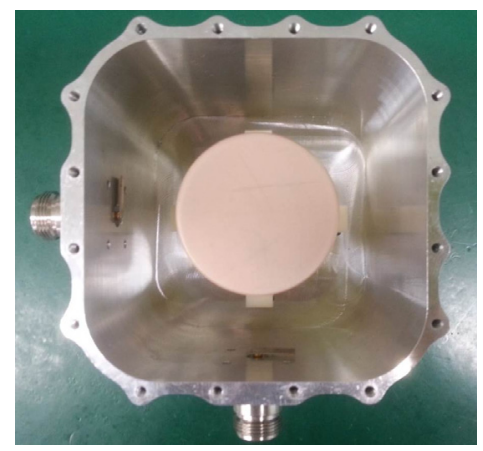

(b)
Fig. 7. Fabricated triple-mode resonators: (a) sphere and (b) rod.

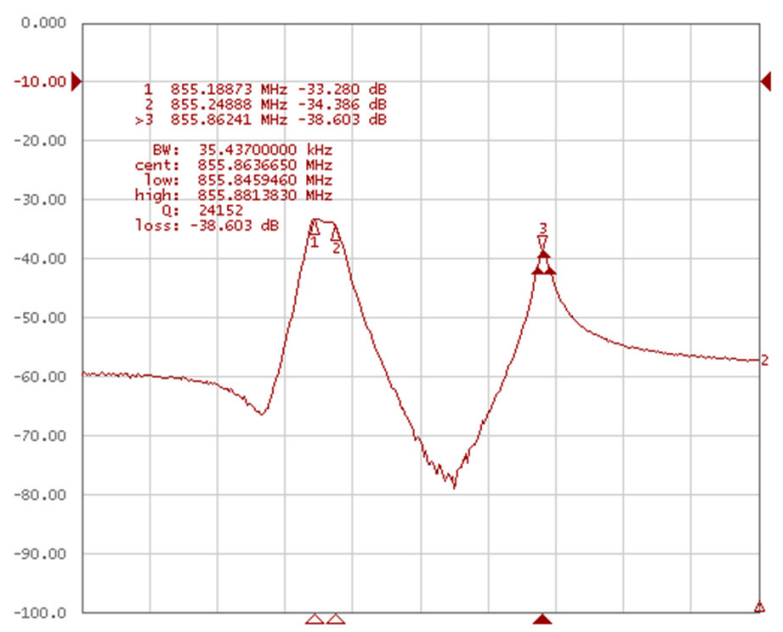

Fig. 8 The measured resonant characteristics of the triple-mode dielectric rod.

\section{Discussion}

The dielectric sphere, as well as the dielectric rod, was fabricated in the metal housing as shown in Fig. 7. The calculated Q values of the resonant dielectric sphere lie between 27,000 and 29,000, depending on the modes, while those of dielectric rod resonator are between 24,000 and 27,000. The quality factors, $Q_{2}$ were measured as in [7]. The measured resonant frequencies and their behaviors are not much different from the simulated results. Measured $Q$ values of the dielectric sphere are over 27,000 depending on the input/output coupling structures within the metal housing. Chamfering the sides of the sphere permits easy separation of the resonant frequencies of $\mathrm{TE}_{01 \delta \mathrm{X}}$ and $\mathrm{TE}_{01 \delta \mathrm{Z}}$ modes. The measured resonant frequencies, including the cross coupling in the dielectric rod resonator, are $855.19 \mathrm{MHz}\left(\mathrm{TE}_{01 \delta \mathrm{X}}\right.$ mode), 855.24 MHz ( $\mathrm{TE}_{01 \text { by }}$ mode), and $855.86 \mathrm{MHz}\left(\mathrm{TE}_{01}\right.$ $\delta z$ mode); the inhomogeneity of the dielectric can introduce a small discrepancy from the simulated results. However, separation of the resonant frequency of degenerate mode $\mathrm{TE}_{01 \delta \mathrm{X}}$ and $\mathrm{TE}_{01 \delta \mathrm{Y}}$ modes for 3-pole bandpass filter applications requires other decoupling schemes. The internal cross coupling can create the transmission zero. For the dielectric rod, the Q values are over 24,000 when it is evaluated

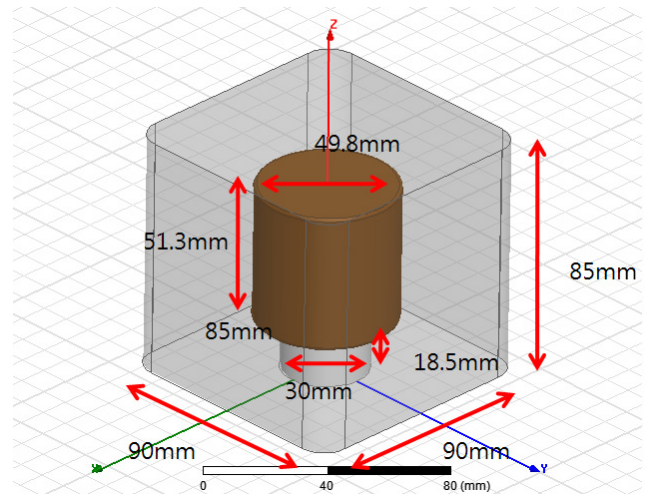

Fig. 9. The dimension of the dielectric rod for the simulation and fabrication.

from the measured results shown in Fig. 8. The dimension of the dielectric rod for the simulation is given in Fig. 9, where the three modes are separated as in Fig. 8. The resonant frequency of the $\mathrm{TE}_{01 \delta \mathrm{Z}}$ mode in the dielectric rod can be moved upward by adjusting its length along the $\mathrm{z}^{-}$ direction compared to its diameter. As is the case for the sphere, separation of the resonant frequency of $\mathrm{TE}_{01 \delta \mathrm{X}}$ mode and $\mathrm{TE}_{01 \delta \mathrm{Y}}$ mode needs other decoupling structures because the separation of the resonant frequency of the degenerate mode is not sufficiently large for the design of a bandpass filter. We obtained very similar behavior in the frequency response for the dielectric sphere as discussed in the simulated results.

\section{CONCLUSION}

Novel triple-mode high-Q spherical dielectric loaded cavity resonators are analyzed for application to bandpass filters for mobile communication systems. Two types of dielectric resonators-spheres and rods-are analyzed and simulated in order to obtain their resonant characteristics. The frequency responses of the two types of resonators behave in the similar way when the resonant frequencies of triple modes are concerned. We also studied the change in the resonant frequencies as a function of the structural parameters of the resonators so that we can use them in the design of bandpass filters.

This research was supported by a grant from the Advanced Technology Center R\&D Program funded by the Ministry of Trade, Industry\& Energy of Korea (No. 10035960).

\section{REFERENCES}

[1] J. F. Liang and W. D. Blair, "High-Q TE01 mode DR filters for PCS wireless base stations," IEEE Transactions on Microwave Theory and Techniques, vol. 46, no. 12, pp. 2493-2500, Dec. 1998.

[2] S. J. Fiedziuszko, "Dual-mode dielectric resonator loaded cavity filters," IEEE Transactions on Microwave Theory 
and Techniques, vol. 30, no. 9, pp. 1311-1316, Sep. 1982.

[3] W. C. Tang, J. Sferrazza, B. Beggs, and D. Siu, "Dielectric resonator output multiplexer for C-band satellite applications," in Proceedings of the IEEE International Microwave Symposium, St. Louis, MO, 1985, pp. 343-345.

[4] V. Walker and I. C. Hunter, "Design of triple-mode TE$01 \delta$ resonator transmission filters," IEEE Microwave and Wireless Components Letters, vol. 12, no. 6, pp. 215-217, Jun. 2002.

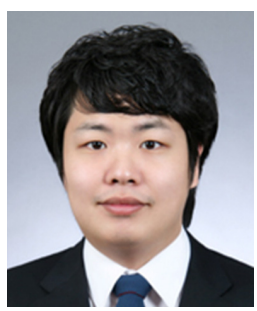

Jae-Yoon Myung received the B.S. degree in electrical engineering from Sogang University in 2013, Seoul, Korea. He is currently working toward a M.S. degree in electronic engineering at Sogang University. His research interests include $\mathrm{RF}$ filters and RF systems.

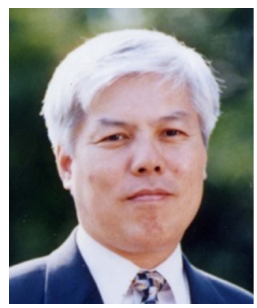

Sang-Won Yun received the B.S. and M.S. degrees in electronic engineering from Seoul $\mathrm{Na}^{-}$ tional University, Seoul, Korea in 1977 and 1979, respectively, and a $\mathrm{Ph} . \mathrm{D}$. degree in electrical engineering from the University of Texas at Austin in 1984. Since 1984, he has been a professor in the department of electronic engineering, Sogang University, Seoul, Korea. Since 2010, he has been a project manager in Korea Communications Commission (KCC). From January 1988 to December 1988, he was a visiting professor at the University of Texas at Austin. Dr. Yun was a president of the Korea Institute of Electromagnetic Engineering and Science (KIEES) in 2007. He was a chairman of the IEEE Microwave Theory and Techniques Society (IEEE MTT-S) Korea Chapter. His research interests include microwave and millimeterwave devices and systems.

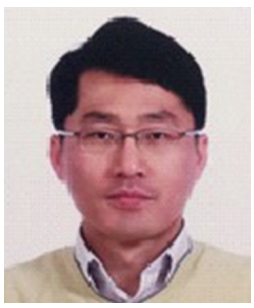

Namshin Park received the B.S. degree in electronic engineering from the Soonchunhayng University, Asan, Korea, and M.S. degree in Management of Technology at Sogang University, Seoul, Korea in 1994 and 2013, respectively. Since 1993, he has been working with the R\&D Center, KMW Inc., Hwasung, Korea, as a RF UNIT group manager. His interests include microwave filter, dielectric resonator, high- $\mathrm{Q}$ and multi-mode resonator and filter miniaturization.
[5] S. Komatsu and Y. Kobayashi, "Design of bandpass filters using triple-mode dielectric rod resonators," Electronics and Communications in Japan (Part II: Electronics), vol. 78, no. 7, pp. 31-40, Jul. 1995.

[6] C. A. Balanis, Advanced Engineering Electromagnetics. New York, NY: John Wiley \& Sons, 1989.

[7] D. Kajfez and P. Guillon, Dielectric Resonators. Dedham, MA: Artech House, 1986.

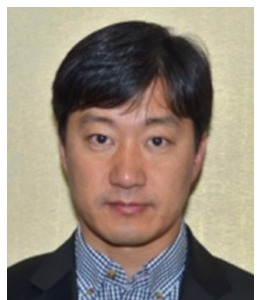

Donyong Lee received the B.S. degree in electronic engineering from the Seoul National University of Science and Technology, Seoul, Korea, in 1998 and is currently working toward the M.S. degree in Management of Technology at Sogang University, Seoul, Korea. Since 1997, he has been working with the R\&D Center, KMW Inc., Hwasung, Korea, as a principal research Engineer. His interests include microwave circuits, cavity and ceramic filters and power handling capability for wireless base station.

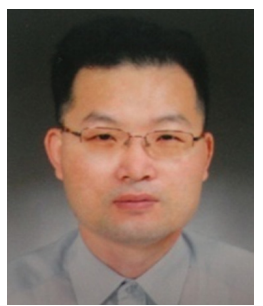

Byungchul Kim received the B.S. degree in electronic engineering from the University of Seoul, Seoul, Korea, in 1992, and is currently working toward the M.S. degree in Management of Technology at Sogang University, Seoul, Korea. Since 1997, he has been working with the R\&D Center, KMW Inc., Hwasung, Korea, as a principal research engineer. His interests include filter related product and radio communication.

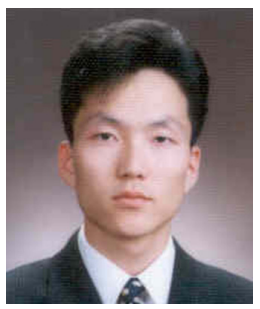

Junghee Won received the B.S. degree in electronic engineering from the Kumoh National Institute of Technology, Gumi, Korea, in 2000. Since 2000, he has been working with the R\&D Center, KMW Inc., Hwasung, Korea, as a principal research engineer. His research interests include microwave cavity and dielectric filter for wireless base stations and co-siting. 


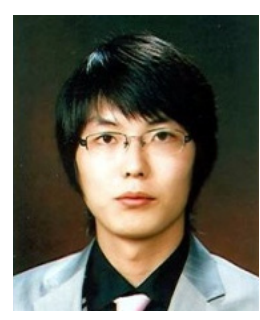

Inho $\mathrm{Na}$ received the B.S. and M.S. degree in electronic engineering from Soonchunhyang University, Asan, Korea, in 2007 and 2009, respectively. In 2012, he joined the R\&D Center, KMW Inc., Hwasung, Korea, as a research engineer. His interests include RF components, front-end devices and small cell base station.

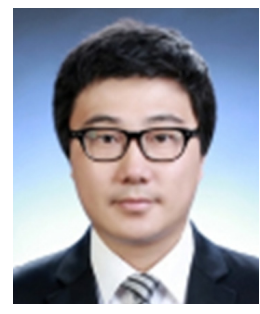

Geonho Jang received the B.S. and M.S. degree in information communication engineering from the University of Incheon (now Incheon National University), Incheon, Korea, in 2009 and 2011, respectively. In 2011, he joined the R\&D Center, KMW Inc., Hwasung, Korea, as a research engineer. His interests include microwave passive components, antennas and metamate-

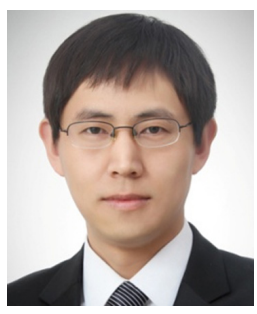

Xu-Guang Wang received the B.Sc. degree in electronic engineering from Qingdao University, Qingdao, China, in 2006, and the Ph.D. degree in electronic engineering from Sogang University, Seoul, Korea, in 2013. In 2013, he joined the R\&D Center, KMW Inc., Korea, where he was involved in developing passive microwave equipment for communication systems. His research interests include RF/microwave filters, and associated passive and active circuits.

rials. 